\title{
Global statistics of liquid water content and effective number concentration of water clouds over ocean derived from combined CALIPSO and MODIS measurements
}

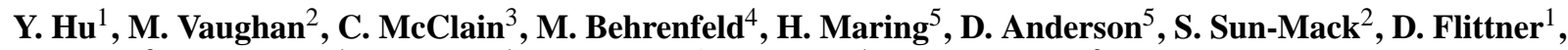 \\ J. Huang ${ }^{2}$, B. Wielicki ${ }^{1}$, P. Minnis ${ }^{1}$, C. Weimer ${ }^{6}$, C. Trepte ${ }^{1}$, and R. Kuehn ${ }^{2}$ \\ ${ }^{1}$ NASA Langley Research Center, Hampton, Virginia, USA \\ ${ }^{2}$ Science Systems and Applications, Inc., Hampton, Virginia, USA \\ ${ }^{3}$ NASA Goddard Space Flight Center, Greenbelt, Maryland, USA \\ ${ }^{4}$ Oregon State University, Corvallis, Oregon, USA \\ ${ }^{5}$ NASA Headquarter, Washington, DC, USA \\ ${ }^{6}$ Ball Aerospace \& Technologies Corp., Builder, Colorado, USA
}

Received: 5 March 2007 - Published in Atmos. Chem. Phys. Discuss.: 28 March 2007

Revised: 5 June 2007 - Accepted: 10 June 2007 - Published: 27 June 2007

\begin{abstract}
This study presents an empirical relation that links the volume extinction coefficients of water clouds, the layer integrated depolarization ratios measured by lidar, and the effective radii of water clouds derived from collocated passive sensor observations. Based on Monte Carlo simulations of CALIPSO lidar observations, this method combines the cloud effective radius reported by MODIS with the lidar depolarization ratios measured by CALIPSO to estimate both the liquid water content and the effective number concentration of water clouds. The method is applied to collocated CALIPSO and MODIS measurements obtained during July and October of 2006, and January 2007. Global statistics of the cloud liquid water content and effective number concentration are presented.
\end{abstract}

\section{Introduction}

In the analysis of polarization-sensitive backscatter lidar data, water clouds provide one of the best understood measurement targets. Their single scattering properties follow Mie theory, and multiple scattering contributions to the signal can be estimated from the depolarization measurements using a simple formula found by $\mathrm{Hu}$ et al. (2006). Nonetheless, the water cloud measurements made by space-based lidars are quite different from those made by passive remote sensing instruments such as MODIS. Passive remote sensing of water clouds, which measures the spectral differences of

Correspondence to: $\mathrm{Y} . \mathrm{Hu}$

(yongxiang.hu-1@nasa.gov) reflected sunlight and thermal emissions, retrieves values of optical depth for the entire vertical column (i.e., on a perpixel basis). Given sufficient sunlight, optical depths can be accurately estimated for values as large as 100 . Passive sensors also provide effective radius information using the absorption in near infrared solar radiation wavelength. Active sensor measurements of water clouds provided by spacebased, dual-polarization, range-resolved vertical profiling lidars provide information about cloud top microphysics to a maximum optical depth of 3 , which, for dense water clouds, corresponds to that portion of the cloud that starts at cloud top and ends at a penetration depth of roughly 100 meters. Unlike reflected sunlight, which consists mostly of multiply scattered photons, the multiple scattering and single scattering contributions to CALIPSO lidar measurements from water clouds are in the same order of magnitude. The multiple scattering contributions are proportional to the number of cloud particles that are within the lidar footprint, and can be separated from the single scattering contributions using the depolarization measurements. Hu and Stamnes (1993) suggested that multiple scattering from water clouds can be well characterized by extinction coefficients and effective radii, and is not sensitive to the variances of droplet size distribution. For passive remote sensing of water clouds using intensity only measurements, the width of size distribution does not impact the water cloud measurements significantly in those cases where multiple scattering dominates the signal. Monte Carlo simulations presented in this study confirm that the insensitivity of multiple scattering to the width of size distribution is also true for most water cloud lidar returns.

Published by Copernicus Publications on behalf of the European Geosciences Union. 


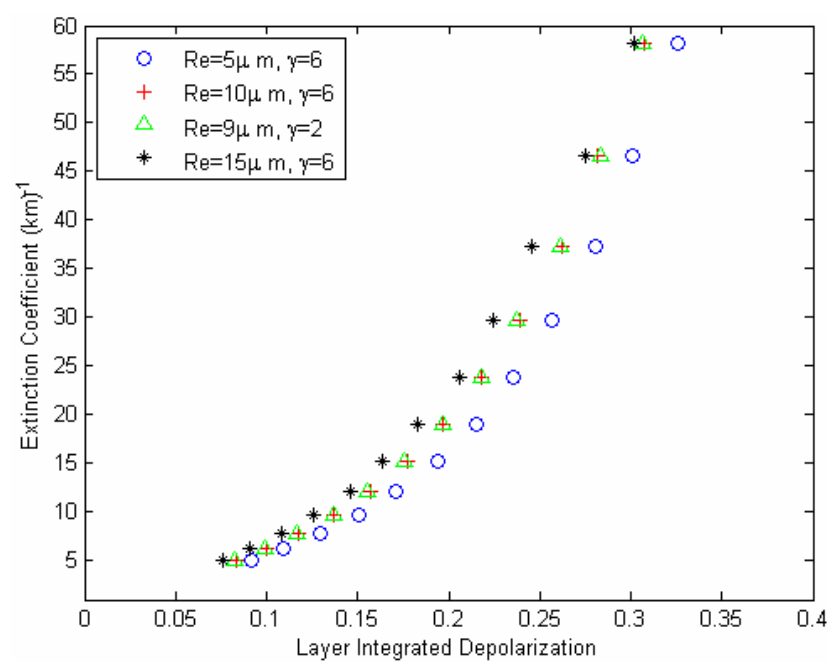

Fig. 1. Theoretical relation between layer averaged extinction coefficients and layer integrated depolarization ratios. Most of the lidar signal comes from the top of the cloud, between optical depths 0 and 2. The extinction coefficient is thus effectively the average extinction coefficient of within $100 \mathrm{~m}$ from cloud top.

Monte Carlo simulations also indicate that, by using layer integrated depolarization ratios and the slope of the exponential decay in the water cloud backscatter due to multiple scattering, both the extinction coefficients and the effective radii of water clouds can be derived from CALIPSO lidar measurements. However, this process would require an accurate deconvolution of the lidar backscatter signal to remove any effects imparted by a non-ideal transient response of the photo-detectors. Because suitable deconvolution techniques are still under development, this study adopts the effective cloud droplet radius retrieved for the Clouds and the Earth's Radiant Energy System (CERES) Project from the Moderate Resolution Imaging Spectral-radiometer (MODIS) (Minnis et al., 1995, 2006). Liquid water content and effective droplet number concentration are then estimated using CALIPSO's lidar depolarization ratio, together with the effective radius derived from the MODIS data.

The purpose of this study includes two aspects:

The first objective is to help establish water clouds as calibration and validation targets for future satellite lidar missions. The global statistics of the water cloud physical properties derived in this study should then provide a baseline for field measurements and ground based water cloud observations. Comparing our derivations with those observations should provide opportunities for the development of better theory and methodology for satellite lidar data analysis of water clouds.

The second objective is to provide the global climate modeling community with an improved global water cloud microphysics climatology that is relevant to understanding ocean-atmosphere fluxes of dimethyl-sulfide and the connec-

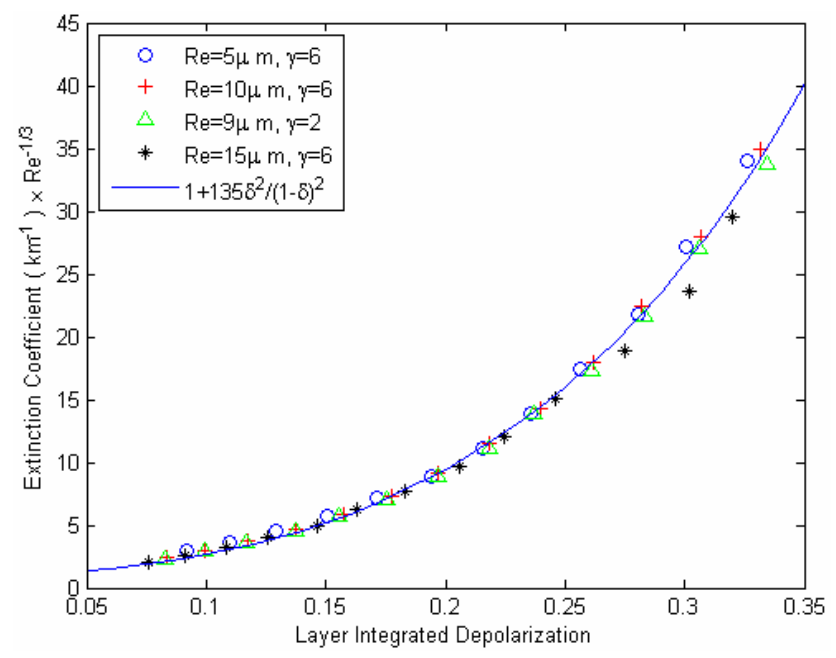

Fig. 2. Relationship between layer-integrated depolarization, extinction coefficient and effective droplet size of water clouds at the CALIPSO viewing geometry. This relation is valid for water clouds with different size distributions and extinction coefficients.

tion with clouds. For a given climate forcing, the climate system may respond in many possible ways. A climate system with a swamp-like surface or a razor thin mixed-layer ocean may respond with fast temperature changes. A climate system with a deep mixed-layer ocean may respond to the same climate forcing with changes in cloud albedo to re-balance the top-of-atmosphere (TOA) radiative fluxes and changes in hydrological cycle to re-balance the surface. Since Shaw (1983) suggested that the interaction between clouds and dimethyl-sulfide $\left(\mathrm{CH}_{3} \mathrm{SCH}_{3}\right.$, DMS $)$ can be an efficient way for the combined ocean and climate system to respond to external forcing, many studies have been conducted in this area (e.g. Charlson et al., 1987; Han et al., 1998). Meanwhile, accurate measurements of biogeochemical processes and cloud microphysics made at suitable spatial and temporal scales remain scarce. Coupled with modeling studies, the water cloud microphysics climatology from combined CALIPSO, MODIS and possibly PARASOL observations will improve the water cloud microphysics observations needed for cloud - climate feedback studies.

\section{A simple and reliable technique for estimating liquid water content and effective droplet number concen- tration from CALIPSO}

As shown in Fig. 1, water cloud extinction coefficients are related to the layer integrated depolarization ratios obtained from the CALIPSO lidar backscatter measurements. This relationship was established via a simulation study using the Monte Carlo code of Hu et al. (2001). Multiple scattering contributions to the backscatter increase as the cloud extinction coefficient increases. Since the depolarization of 


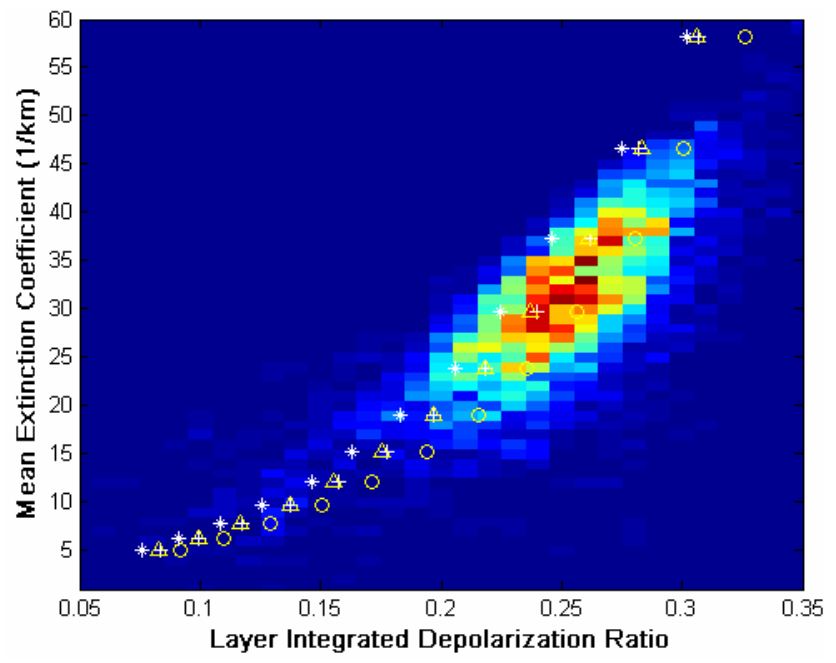

Fig. 3. Histogram of water cloud extinction coefficient and depolarization ratio relation from CALIPSO data. The extinction values are retrieved using CALIPSO vertical profiles. The markers are the modeling results of Fig. 1.

radiance from water clouds is proportional to multiple scattering, the depolarization ratio increases for denser clouds as well. For smaller particles, when contrasted with large particles, both multiple scattering and depolarization ratio increase faster with extinction coefficient, since bigger particles scatter more in the forward direction, thus reducing the chances of backscatter. The width of droplet size distribution has minimal impact on the extinction coefficient - depolarization relation.

An interesting empirical relation among the extinction coefficient $\beta$, the effective radius $R_{e}$ and the layer-integrated depolarization ratio $\delta$, illustrated in Fig. 2, is

$\beta\left(\frac{R_{e}}{R_{e 0}}\right)^{-1 / 3}=1+135 \frac{\delta^{2}}{(1-\delta)^{2}}$.

This relation is derived from Monte Carlo simulations that incorporate the CALIPSO instrument specifications, viewing geometry, and footprint size. The unit of $R_{e}$ is in $\mu \mathrm{m}$ and $\beta$ is in $\mathrm{km}^{-1} . R_{e 0}$ is $1 \mu \mathrm{m}$. Both coefficients ( 1 and 135) have units of $\mathrm{km}^{-1}$. The CALIPSO receiver has channels which separately measure the backscattered signal in orientations parallel and perpendicular to the polarization plane of the outgoing, linearly polarized laser pulse. The layer-integrated depolarization ratio is then formed by dividing the integrated attenuated backscatter from the perpendicular channel by the integrated attenuated backscatter from the parallel channel.

Ideally, the value of $\beta$ will be derived from CALIPSO water cloud measurements using the exponential decay of water cloud attenuated backscatter $\gamma^{\prime}$ with range $r$ within the clouds, $\gamma^{\prime}=\gamma_{0} e^{-2 \eta \beta r}$. After de-convolution, the slope of the exponential decay of the water-cloud attenuated backscatter, $\eta \beta$, can, in practice, be obtained using 4 range bins under-
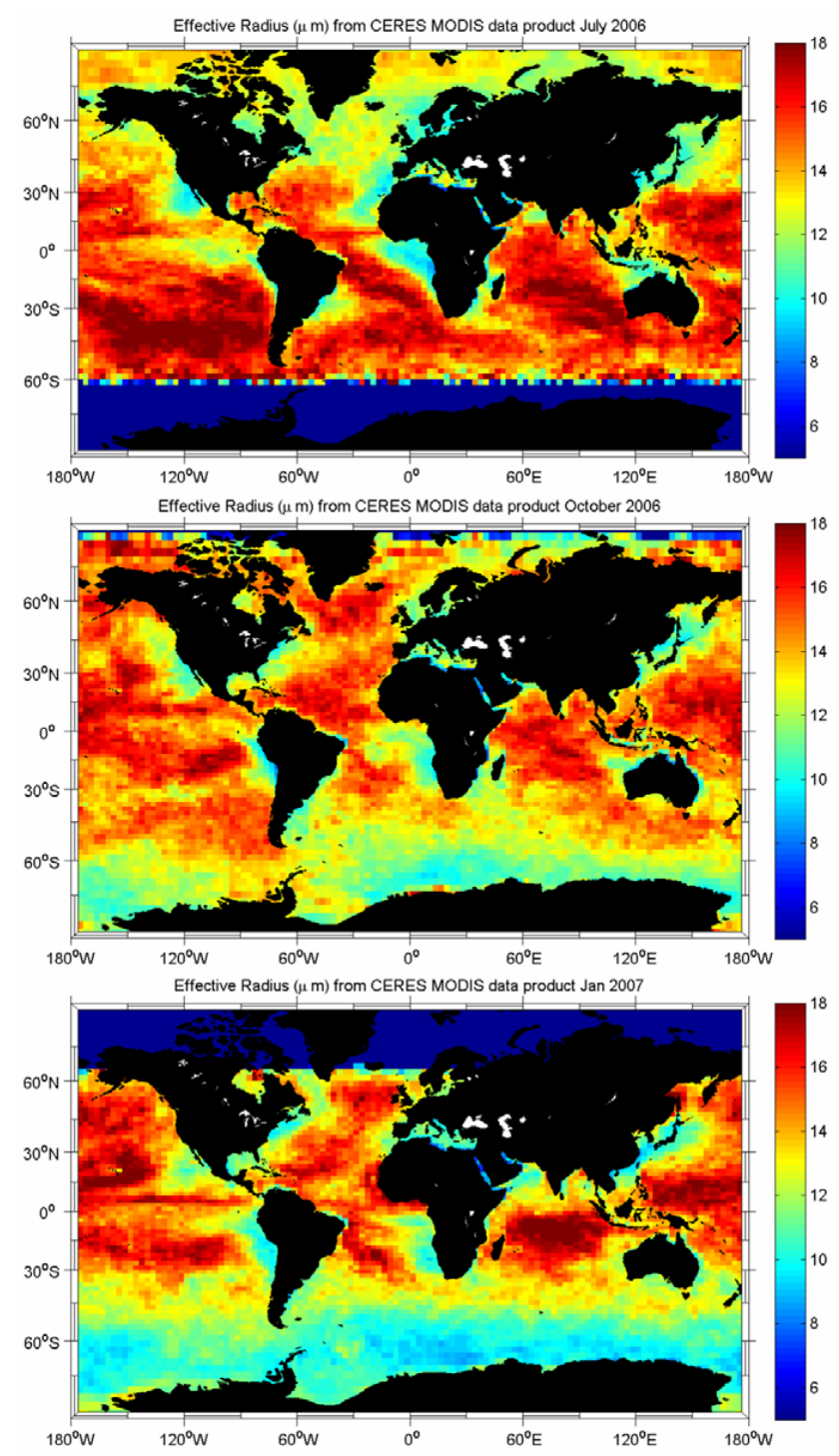

Fig. 4. Monthly mean water cloud effective radii from CERES MODIS cloud product.

neath the peak of the water cloud lidar return. Thus the cloud top extinction coefficient $\beta$ can be derived by applying the simple relation between multiple scattering factor $\eta$ from $\mathrm{Hu}$ et al. (2007),

$\eta=\left(\frac{1-\delta}{1+\delta}\right)^{2}$.

In Fig. 3, the scatter plot of the extinction coefficients derived from CALIPSO data versus the corresponding CALIPSO depolarization ratio measurements is seen to be very similar to the relationship developed from the Monte Carlo simulation results in Fig. 1.

After retrieving the extinction coefficient, we can apply it to Eq. (1) to derive $R_{e}$ from the depolarization ratio measurements. The de-convolution process, which removes the 

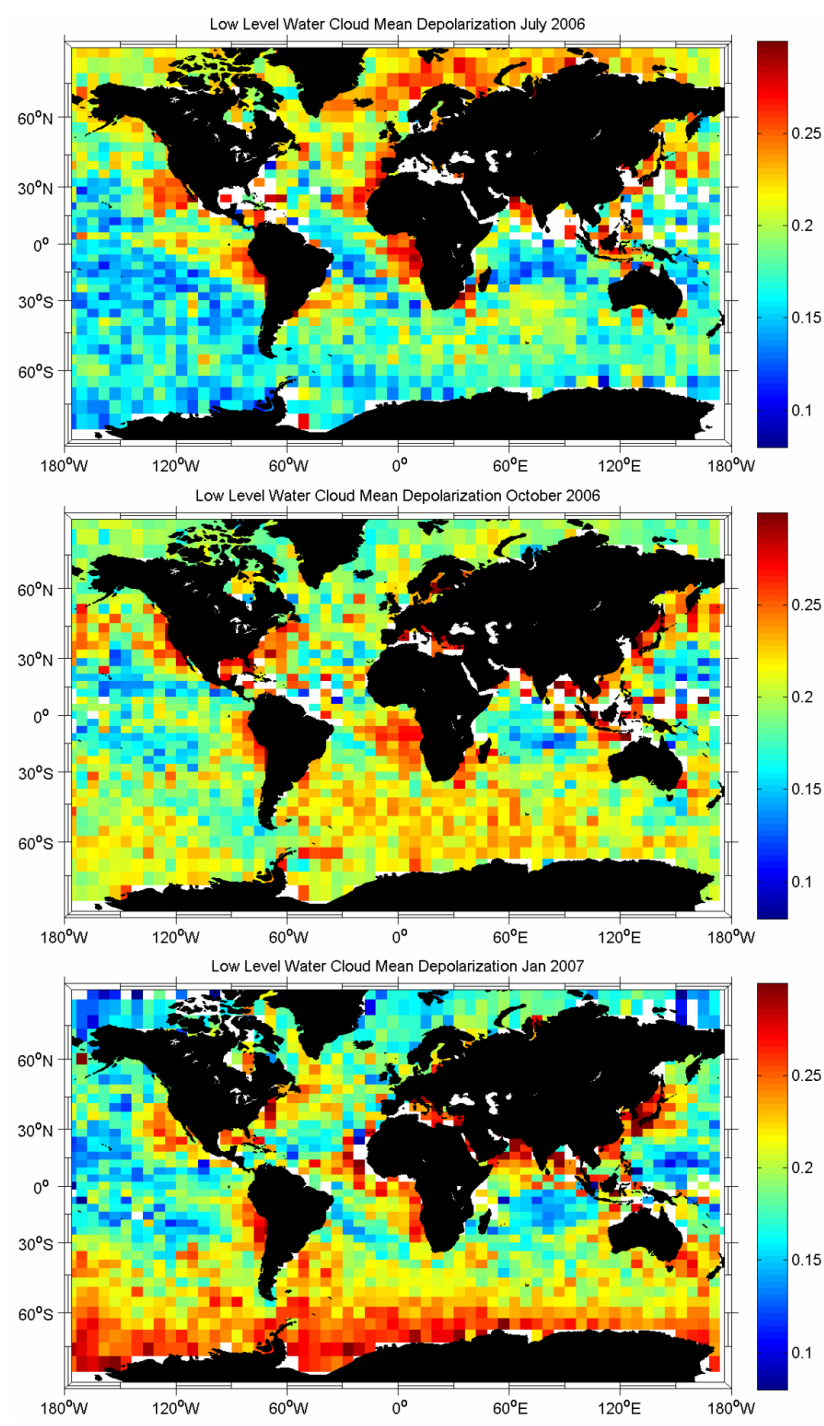

Fig. 5. Monthly mean depolarization of low level water clouds.

tail of the lidar receiver's transient response function, depends on the response function used and on the exact cloud top location within the $30 \mathrm{~m}$ range bin, can introduce errors and may not always be stable. These errors in the extinction coefficient will be magnified when it is subsequently used for deriving effective radius using Eq. (1). Because of these caveats, this approach can, at present, only be used for research purposes with limited case studies. We will be revisiting it in the future for CALIPSO water data analyses after the instrument transient response is well characterized.

As an alternative approach for deriving extinction coefficient and effective droplet radius, we adopted the collocated water cloud droplet sizes retrieved from MODIS 3.7- $\mu \mathrm{m}$ data for CERES (Minnis et al., 2006). The number of photons scattered into the forward direction increases with particle size. Thus, the chance of the photon being absorbed at the
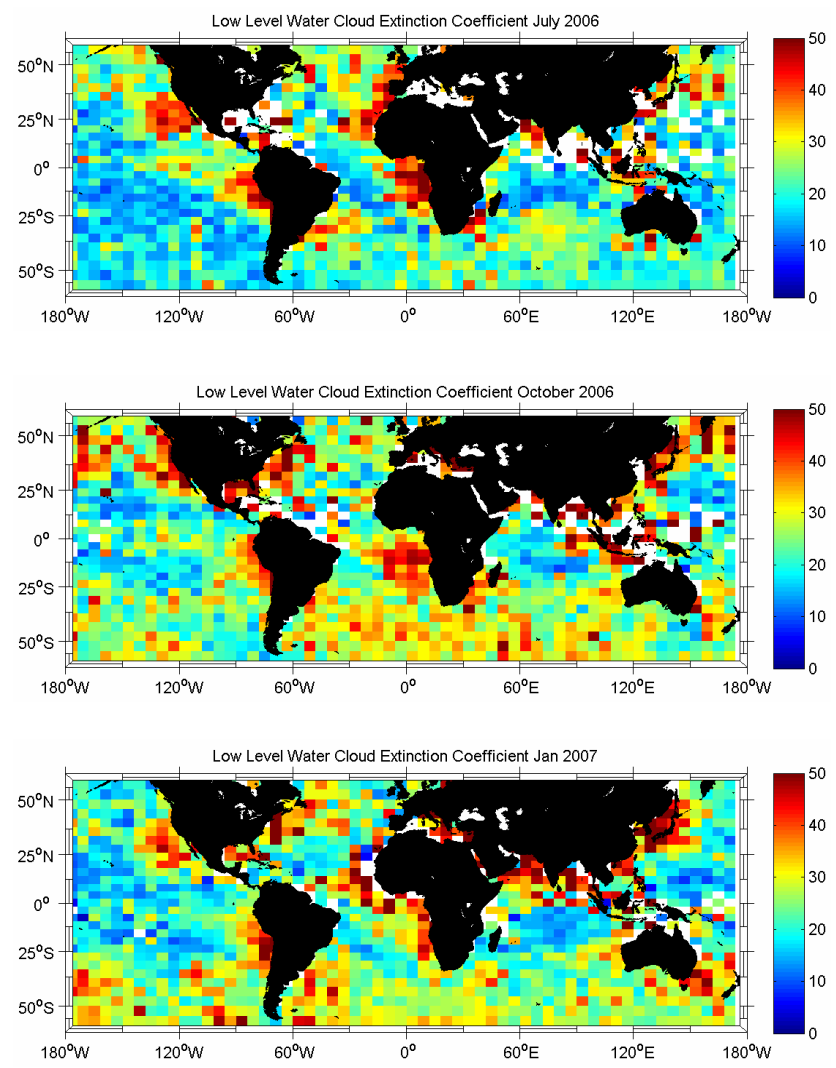

Fig. 6. Monthly mean water cloud extinction coefficient $(1 / \mathrm{km})$ at various longitude/latitude boxes.

near-infrared wavelengths before returning back to space increases with size. For the same optical depths, water clouds with larger droplets are darker in the near-infrared wavelengths. The effective droplet radius derived from the absorption at $3.7 \mu \mathrm{m}$ reflects the average size information from the very top part of water clouds, with a vertical penetration depth similar to the CALIPSO lidar signal. Figure 4 shows the global distributions of the monthly mean Re from the Aqua CERES-MODIS analysis of July 2006, October 2006 and January 2007.

Using the layer integrated depolarization ratios (Fig. 5) measured in water clouds, together with the coincident $R_{e}$ from CERES-MODIS cloud retrievals, we can derive the extinction coefficients $\beta$ and liquid water content LWC of the water clouds,

$$
\begin{aligned}
& \beta=\left(\frac{R_{e}}{R_{e 0}}\right)^{1 / 3}\left\{1+135 \frac{\delta^{2}}{(1-\delta)^{2}}\right\}, \\
& \mathrm{LWC} \approx \frac{2 \rho R_{e} \beta}{3}=\frac{0.002 R_{e}}{3}\left(\frac{R_{e}}{R_{e 0}}\right)^{1 / 3}\left\{1+135 \frac{\delta^{2}}{(1-\delta)^{2}}\right\} .
\end{aligned}
$$

Here, LWC is given in $\mathrm{g} / \mathrm{m}^{3}$ and $R_{e}$ in $\mu \mathrm{m} . \rho$ is the density of liquid water, expressed in $\mathrm{g} / \mathrm{cm}^{3} . R_{e 0}$ is $1 \mu \mathrm{m}$. Figures 6 and 7 show the monthly mean extinction coefficients and liquid water contents computed from the collocated CALIPSO 

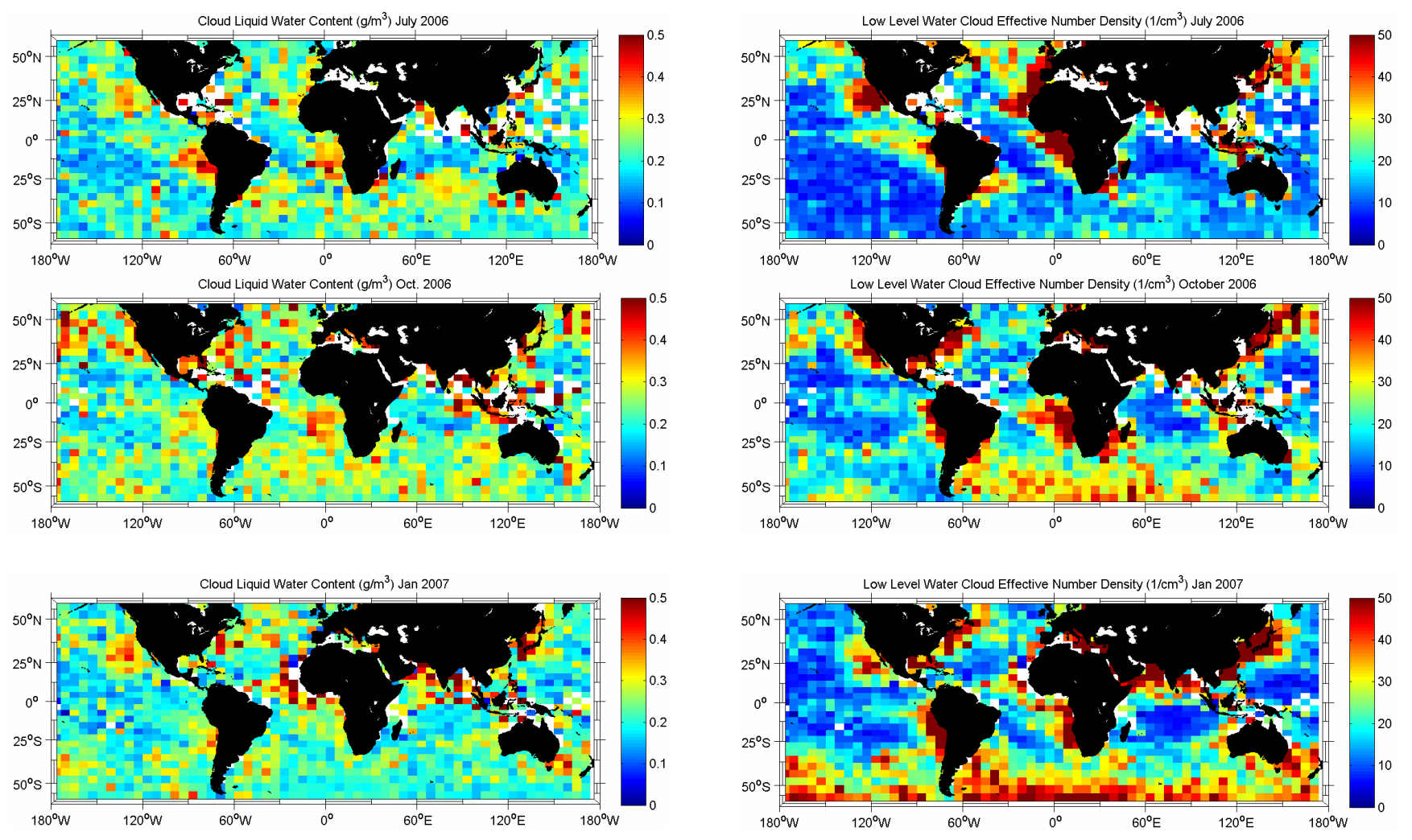

Fig. 7. Monthly mean liquid water content of low-level water clouds.

depolarization and MODIS effective droplet sizes, respectively. The liquid water content values agree with various historical in situ measurements, e.g. the aircraft-based Gerber probe measurements at the South Ocean Cloud Experiment off the western coast of Tasmania by Gerber et al. (2001).

For water clouds with a mono-disperse droplet size distribution, the water cloud particle number concentration $N$ can be relatively accurately estimated if the extinction coefficient is known,

$N_{\text {mono }}=\frac{\beta}{2 \pi r_{e}^{2}}$.

In order to derive particle number concentration $N$ of water clouds with various sizes, an assumption has to be made about the shape of the size distributions. Here we assume a generalized gamma distribution (Hu et al., 1993; Miles et al., 2000),

$n(r)=\frac{N}{\Gamma(\gamma) r_{m}}\left(\frac{r}{r_{m}}\right)^{\gamma-1} \exp \left(-r / r_{m}\right)$.

Here $(\gamma-1) r_{m}$ is the mode radius of the size distribution, and $\gamma$ is the parameter representing the width of the size distributions (the larger the $\gamma$ is, the narrower the size distribution). $\frac{1}{\gamma+2}$ is the effective variance using the definition of Hansen

Fig. 8. Monthly mean effective droplet number concentration of water clouds.

(1971). The particle number concentration and extinction coefficient can be approximately related as,

$\beta=2 \pi N \overline{r^{2}}=2 \pi \int n(r) r^{2} d r=2 \pi N(\gamma+1) \gamma r_{m}^{2}$,

$R_{e}=\int n(r) r^{3} d r / \int n(r) r^{2} d r=(\gamma+2) r_{m}$,

$N=\frac{\beta}{2 \pi(\gamma+1) \gamma r_{m}^{2}}=\frac{\beta}{2 \pi R_{e}^{2}} \frac{(\gamma+2)^{2}}{(\gamma+1) \gamma}=N_{e} \frac{(\gamma+2)^{2}}{(\gamma+1) \gamma}$.

The effective number concentration, $N_{e}=\frac{\beta}{2 \pi R_{e}^{2}}$, can be derived from depolarization ratios and $R_{e}$ following Eq. (3),

$N_{e}=1000 \frac{1+135 \delta^{2} /\left(1-\delta^{2}\right)}{2 \pi\left(\frac{R_{e}}{R_{e 0}}\right)^{5 / 3}}$.

The effective number concentration $N_{e}$ is expressed in $\mathrm{cm}^{-3}$. $R_{e 0}$ is once again $1 \mu \mathrm{m}$. Compared to the true droplet number concentration $N$, the effective number concentration is more relevant to absorption in the near infrared and thus to the cloud albedo. However, $N_{e}$ is not as closely related to cloud condensation nucleii (CCN) as the true droplet number concentration. 
From Eq. (8), $N_{e} / N=\gamma(\gamma+1) /(\gamma+2)^{2}$, and thus the effective droplet number concentration $N_{e}$ and the true droplet number concentration $N$ are very close when $\gamma$ is large enough (i.e., when the effective variance is small enough). The difference between $N$ and $N_{e}$ is less than $50 \%$ whenever $\gamma$ is greater than 3 (effective variance smaller than 0.2), as is the case for most water clouds. The difference can be accounted for using information about the width of the droplet size distribution. The effective number concentration $N_{e}$ is the same as the true number concentration $N$ for very narrow size distributions and thus very large $\gamma$ values, where $\frac{(\gamma+1) \gamma}{(\gamma+2)^{2}} \approx 1$. In general, the effective water cloud droplet number concentration is less than the true number concentration and the difference increases with the width of the size distribution.

Because the effective variance of the gamma distribution is $\frac{1}{\gamma+2}$, values of $\gamma>10$ require the variance of the gamma distribution to be less than 0.08. In an analysis of the angular pattern of the linear polarization from the POLDER measurements, Breon and Goloub (1998) suggest that the variance of water cloud droplet size distribution can be as small as 0.02 . For water clouds with a 0.02 variance in size distribution, $N_{e}=0.94 \mathrm{~N}$. For water clouds with a 0.1 variance in size distribution, $N_{e}=0.72 N$. Miles et al. (2000) compiled all the available aircraft in situ measurements of water clouds and found the largest variance is as high as 0.2. Any knowledge of the width will help make that difference smaller. The CERES-MODIS retrievals use a modified gamma distribution having an effective variance of 0.1 (Minnis et al., 1998).

Figure 8 shows the global and seasonal distribution of the water cloud effective number concentration. The true water cloud droplet number concentration can be estimated from Eq. (8) using climatological values of size distribution widths. The errors in the number concentration can be assessed using the size distribution width information retrieved from polarization measurements of the glory scattering angle and rainbow scattering angles.

\section{Discussion}

Using depolarization ratios provided directly by CALIPSO, together with effective radius values obtained from MODIS measurements, we can derive the effective cloud particle number concentration of water clouds. When the width of the cloud droplet size distribution is known, the true number concentration can be accurately estimated from the effective number concentration. Statistics of the true water cloud particle number concentration will be compiled in the future, using a climatology of size distribution widths estimated from rainbow and glory information obtained from PARASOL data, as well as other climatological values of size distributions from ground and in situ measurements.

The spatial and seasonal variations of water cloud effective droplet number concentration derived from this study show some similarity to ocean biogeochemistry processes, and in general agree with the patterns of DMS concentration seasonal and temporal variations in the middle and low latitudes, generated from the POP Ocean GCM by Chu et al. (2004). Further similarities are expected between the seasonal and spatial variations of $N_{e}$ and the ocean primary productivity and phytoplankton, especially at middle to high latitudes, consistent with the observations of Meskhidze et al. (2006).

The ocean mixed-layer depth change and corresponding changes in low level water cloud amount may be as efficient in terms of re-establishing the balance of TOA and surface radiative fluxes as the sulfur cycle and cloud microphysics hypotheses suggested by Shaw (1983). Both require more studies using combined active and passive remote sensing of the ocean and the atmosphere.

Edited by: Qiang Fu

\section{References}

Breon, F. M. and Goloub, P.: Cloud droplet, effective radius from spaceborne polarization. measurements, Geophys. Res. Lett., 25, 1879-1992, 1998.

Charlson, R. J., Lovelock, J. E., Andreae, M. O., and Warren, S. G.: Oceanic phytoplankton, atmospheric sulfur, cloud albedo and climate, Nature, 326, 655-661, 1987.

Chu, S., Elliott, S., and Maltrud, M.: Ecodynamic and EddyAdmitting Dimethyl Sulfide Simulations in a Global Ocean Biogeochemistry/Circulation Model, Earth Interact., 8, 11, doi:10.1175/1087-3562, 2004.

Gerber, H., Jensen, J. B., Davis, A. B., Marshak, A., and Wiscombe, W. J.: Spectral Density of Cloud Liquid Water Content at High Frequencies, J. Atmos. Sci., 58, 497-503, 2001.

Han, Q., Rossow, B., Chou, J., and Welch, R.: Global Survey of the Relationships of Cloud Albedo and Liquid Water Path with Droplet Size Using ISCCP, J. Climate, 11, 1616-1528, 1998.

Hanson, J.: Multiple Scattering of Polarized Light in Planetary Atmospheres, J. Atmos. Sci., 28, 1400-1426, 1971.

Hu, Y. and Stamnes, K.: An accurate parameterization of cloud radiative properties suitable for climate models, J. Climate, 6, 728742, 1993.

Hu, Y., Winker, D., Yang, P., Baum, B., L. Poole, and L. Vann: Identification of cloud phase from PICASSO-CENA lidar depolarization: A multiple scattering sensitivity study, J. Quant. Spectros. Radiat. Trans., 70, 569-579, 2001.

Hu, Y., Liu, Z., Winker, D., Vaughan, M., Noel, V., Bissonnette, L., Roy, G., and McGill, M.: A simple relation between lidar multiple scattering and depolarization for water clouds, Opt. Lett., 31, 1809-1811, 2006.

Meskhidze, N. and Nenes, A.: Photoplankton and cloudness in the southern ocean, Science, 314, 1919, 2006.

Miles, N. J., Verlinde, J., and Clothiaux, E. E.: Cloud droplet size distributions in low-level stratiform clouds, J. Atmos. Sci., 57, 295-311, 2000.

Minnis, P., Kratz, D. P., Coakley Jr., J. A., et al.: Cloud Optical Property Retrieval (Subsystem 4.3), Clouds and the Earth's Radiant Energy System (CERES) Algorithm Theoretical Basis 
Document, Volume III: Cloud Analyses and Radiance Inversions (Subsystem 4), NASA RP 1376 Vol. 3, edited by: CERES Science Team, December, 135-176, 1995.

Minnis, P., Garber, D. P., Young, D. F., Arduini, R. F., and Takano, Y.: Parameterization of reflectance and effective emittance for satellite remote sensing of cloud properties, J. Atmos. Sci., 55, 3313-3339, 1998.
Minnis, P., Geier, E., Wielicki, B., et al.: Overview of CERES cloud properties from VIRS and MODIS, Proc. AMS 12th Conf. Atmos. Radiation, Madison, WI, July 10-14, CD-ROM, J2.3., 2006.

Shaw, G.: Aerosols as climate regulators: a climate-biosphere linkage, Atmos. Environ., 21, 985-986, 1983. 